\title{
ARTICLE
}

Minimal resdiual disease

\section{Moxetumomab pasudotox in relapsed/refractory hairy cell leukemia}

\author{
Robert J. Kreitman ${ }^{1} \cdot$ Claire Dearden $^{2} \cdot$ Pier Luigi Zinzani $^{3} \cdot$ Julio Delgado $^{4} \cdot$ Lionel Karlin $^{5} \cdot$ Tadeusz Robak $^{6}$. \\ Douglas E. Gladstone $\mathbb{D}^{7}$. Philipp le Coutre ${ }^{8}$. Sascha Dietrich ${ }^{9} \cdot$ Mirjana Gotic $\mathbb{D}^{10}$ - Loree Larratt ${ }^{11}$. Fritz Offner $^{12}$. \\ Gary Schiller $^{13} \cdot$ Ronan Swords $^{14} \cdot$ Larry Bacon $^{15} \cdot$ Monica Bocchia $^{16} \cdot$ Krimo Bouabdallah $^{17}$ - Dimitri A. Breems ${ }^{18}$. \\ Agostino Cortelezzi ${ }^{19} \cdot$ Shira Dinner ${ }^{20} \cdot$ Michael Doubek $^{21} \cdot$ Bjorn Tore Gjertsen $\mathbb{D}^{22} \cdot$ Marco Gobbi $^{23}$. \\ Andrzej Hellmann ${ }^{24}$. Stephane Lepretre ${ }^{25}$ - Frederic Maloisel ${ }^{26}$. Farhad Ravandi ${ }^{27}$. Philippe Rousselot ${ }^{28,29}$.

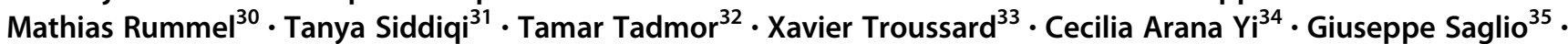 \\ Gail J. Roboz ${ }^{36} \cdot$ Kemal Balic $^{37} \cdot$ Nathan Standifer $^{37} \cdot$ Peng He $^{38}$. Shannon Marshall ${ }^{38} \cdot$ Wyndham Wilson $^{1} \cdot$ \\ Ira Pastan ${ }^{1} \cdot$ Nai-Shun $\mathrm{Yao}^{38} \cdot$ Francis Giles ${ }^{39}$
}

Received: 6 June 2018 / Accepted: 8 June 2018 / Published online: 20 July 2018

(c) The Author(s) 2018. This article is published with open access

\begin{abstract}
This is a pivotal, multicenter, open-label study of moxetumomab pasudotox, a recombinant CD22-targeting immunotoxin, in hairy cell leukemia (HCL), a rare B cell malignancy with high CD22 expression. The study enrolled patients with relapsed/ refractory HCL who had $\geq 2$ prior systemic therapies, including $\geq 1$ purine nucleoside analog. Patients received moxetumomab pasudotox $40 \mu \mathrm{g} / \mathrm{kg}$ intravenously on days 1,3 , and 5 every 28 days for $\leq 6$ cycles. Blinded independent central review determined disease response and minimal residual disease (MRD) status. Among 80 patients (79\% males; median age, 60.0 years), durable complete response (CR) rate was 30\%, CR rate was $41 \%$, and objective response rate (CR and partial response) was 75\%; 64 patients (80\%) achieved hematologic remission. Among complete responders, $27(85 \%)$ achieved MRD negativity by immunohistochemistry. The most frequent adverse events (AEs) were peripheral edema (39\%), nausea (35\%), fatigue (34\%), and headache (33\%). Treatment-related serious AEs of hemolytic uremic syndrome (7.5\%) and capillary leak syndrome (5\%) were reversible and generally manageable with supportive care and treatment discontinuation (6 patients; 7.5\%). Moxetumomab pasudotox treatment achieved a high rate of independently assessed durable response and MRD eradication in heavily pretreated patients with HCL, with acceptable tolerability.
\end{abstract}

\section{Introduction}

Patients with hairy cell leukemia (HCL), a rare B cell malignancy characterized by high CD22 expression, typically present with pancytopenia and increased susceptibility to infection [1]. Although many patients achieve long-term complete remission with the purine nucleoside analogs pentostatin or cladribine [2-4], $\sim 50 \%$ will relapse by 16

Results were presented in part at the ASCO Annual Meeting in Chicago, IL, June 1-5, 2018.

Electronic supplementary material The online version of this article (https://doi.org/10.1038/s41375-018-0210-1) contains supplementary material, which is available to authorized users.

Francis Giles

frankgiles@aol.com

Extended author information available on the last page of the article. years and require additional treatment [5]. In later lines, purine nucleoside analogs offer lower complete response rate, shorter duration of response, and higher risk of cumulative toxicity compared with earlier treatment [5-7]. Of note, purine nucleoside analogs are associated with severe infection due to profound neutropenia [8]. Targeted therapies, such as vemurafenib, ibrutinib, and rituximab, show encouraging efficacy but rarely eradicate minimal residual disease in patients with complete response as single agents, and are often associated with safety and tolerability concerns [5, 9-18]. An unmet need remains in relapsed/refractory HCL for therapies that provide durable complete response with eradication of minimal residual disease and less myelo/ immunosuppression.

Moxetumomab pasudotox (CAT-8015) is a recombinant immunotoxin targeting CD22, composed of an immunoglobulin light chain variable domain and a heavy chain variable domain genetically fused to a truncated form of 
Pseudomonas exotoxin PE38 [19]. In a phase 1 study of patients with relapsed/refractory HCL, 49 patients received moxetumomab pasudotox [20]. The objective response rate was $86 \%$, with a complete response rate of $57 \%$; $63 \%$ of patients who achieved complete response were minimal residual disease negative by immunohistochemistry as assessed by an independent central reviewer, and similar percentage by flow cytometry, either of which translated into longer duration of complete response and/or progression-free survival $[20,21]$. The current study evaluated the rate of durable complete response with moxetumomab pasudotox in patients with multiply relapsed HCL.

\section{Methods}

\section{Study design and organization}

This pivotal, multicenter, single-arm open-label study (clinicaltrials.gov identifier: NCT01829711) was conducted at 32 centers in 14 countries. The study was performed in accordance with the principles of the Declaration of Helsinki, the International Conference on Harmonisation/Good Clinical Practice guidelines, and applicable regulatory requirements. The protocol was approved by the institutional review board at each center. All patients provided written informed consent.

The authors designed the study in collaboration with the sponsor. Data were collected and analyzed by the sponsor and interpreted jointly with the authors. All authors had full access to the data. The first draft was written by the authors and Peloton Advantage, who also provided editorial support. All authors reviewed and contributed to subsequent drafts and vouch for the completeness and veracity of the data and analyses, and for adherence to the protocol.

\section{Patients}

Adults with histologically confirmed HCL and an indication for treatment (defined as at least one of the following: neutrophils $<1.0 \times 10^{9} / \mathrm{L}$, platelets $<100 \times 10^{9} / \mathrm{L}$, hemoglobin $<10 \mathrm{~g} / \mathrm{dL}$, or symptomatic splenomegaly) were eligible. Patients must have received at least two prior systemic therapies, including two courses of a purine nucleoside analog or one course of rituximab or a $B R A F$ inhibitor following a single prior purine nucleoside analog course. An Eastern Cooperative Oncology Group performance status of 0,1 , or 2 and adequate hepatic and renal function were required. Detailed inclusion and exclusion criteria are shown in the protocol.

\section{Study treatment}

Patients received moxetumomab pasudotox $40 \mu \mathrm{g} / \mathrm{kg}$ intravenously over 30 minutes on days 1,3 , and 5 of 28-day cycles for a maximum of six cycles or until documentation of minimal residual disease-negative complete response (as assessed by the investigator), disease progression, initiation of alternate therapy, or unacceptable toxicity. Patients received prophylaxis for renal insufficiency (fluids and lowdose aspirin) and hypersensitivity reactions (hydroxyzine, acetaminophen, and ranitidine; see protocol for details).

\section{Study end points and assessments}

The primary end point was durable complete response, defined as complete response assessed by blinded independent central review with maintenance of hematologic remission for more than 180 days. Complete response was defined based on pathology (no evidence of hairy cells in bone marrow by routine hemoxylin and eosin stain), imaging (resolution of splenomegaly, hepatomegaly, and lymphadenopathy, documented by CT or MRI), and normalization of hematologic parameters (neutrophils $\geq 1.5 \times 10^{9} / \mathrm{L}$, platelets $\geq$ $100 \times 10^{9} / \mathrm{L}$, and hemoglobin $\geq 11.0 \mathrm{~g} / \mathrm{dL}$ without growth factors or transfusions in 4 weeks). Relapse was defined as loss of any criteria needed for best response, including asymptomatic reappearance of hairy cells in the bone marrow by hemoxylin and eosin stain. Additional details are described in the protocol. Secondary efficacy end points included objective response rate, duration of complete and objective response, progression-free survival, safety/tolerability, immunogenicity, and pharmacokinetics.

Minimal residual disease was independently assessed using immunohistochemistry. A blinded independent pathologist assessed bone marrow biopsy specimens stained for the HCL/B cell antigens CD20, CD79a, Annexin A1, DBA.44, and PAX-5; additional details are in the Supplementary Appendix. During treatment, minimal residual disease was assessed locally at study sites by flow cytometric analysis of peripheral blood and/or bone marrow aspirate, according to each site's procedures.

Safety assessments included adverse events, serious adverse events, and changes in clinical laboratory evaluations and vital signs through 30 days after the last moxetumomab pasudotox dose. Adverse events and serious adverse events were assessed by the investigators for relationship to moxetumomab pasudotox, graded using National Cancer Institute Common Toxicity Criteria for Adverse Events V4.03 and coded using Medical Dictionary for Regulatory Activities V20.0.

Plasma moxetumomab pasudotox concentrations were assessed at multiple time points following dosing. 
Pharmacokinetic parameters were estimated by noncompartmental approach using Phoenix ${ }^{\circ}$ WinNonlin ${ }^{\circ}$ (Version 6.3, Certara, Princeton, New Jersey). Immunogenicity was evaluated at multiple time points. Samples that were positive for anti-drug antibodies were evaluated for neutralization, specificity (PE38 versus CD22 binding domain), and titer. Pharmacodynamics were assessed by measuring peripheral blood B cell counts $(\mathrm{CD} 19+\mathrm{B}$ cells which include hairy cells) at multiple time points. Further details are provided in the protocol.

Efficacy was evaluated in the intent-to-treat population, which included all patients who entered and received moxetumomab pasudotox, and safety was evaluated in the safety population, which comprised all patients who received at least one dose of moxetumomab pasudotox; both populations comprise the same 80 patients.

\section{Statistical analysis}

Rituximab was used as a historical control to determine sample size, as it was the most frequently used nonchemotherapy treatment in relapsed/refractory HCL with a $\mathrm{CR}$ rate of $13 \%$ in the largest study [12, 22]. A sample size of 77 patients was planned to provide $90 \%$ power to detect a difference between $13 \%$ and $28 \%$ in durable complete response rates using a 2-sided significance level of 0.05 . Durable complete response rate was constructed using the exact probability method (Clopper-Pearson exact interval). If the lower bound of the $95 \%$ CI was above $13 \%$ (or equivalently, the binomial exact test one-sided $p$-value $<0.025$ ), it is concluded that the durable complete response rate was significantly higher than the historical control value of $13 \%$.

Duration of complete response, duration of objective response, and progression-free survival were estimated with the use of the Kaplan-Meier method. In the analysis of duration of complete response and objective response, patients alive with no documented relapse prior to data cutoff, dropout, or initiation of alternative anticancer therapy were censored on the date of last disease assessment or hematologic assessment, whichever occurred last. In the analysis of progression-free survival, patients alive with no documented relapse or disease progression prior to data cutoff, dropout, or the initiation of alternative anticancer therapy were censored on the date of last disease assessment or hematologic assessment, whichever occurred last.

\section{Role of funding source}

This study and manuscript were funded by MedImmune, the global biologics R\&D arm of AstraZeneca. MedImmune employees were involved in the study design, the collection, analysis, and interpretation of data, the review of the manuscript, and the decision to submit for publication.
Table 1 Demographics and baseline characteristics

\begin{tabular}{|c|c|}
\hline Characteristic & Value $(N=80)$ \\
\hline \multicolumn{2}{|l|}{ Age } \\
\hline Median, $y$ & 60.0 \\
\hline Range, $y$ & $34-84$ \\
\hline \multicolumn{2}{|l|}{ Race (excluding patients enrolled in France $[n=8]$} \\
\hline White, $n(\%)$ & $70(97.2)$ \\
\hline Black, $n(\%)$ & $1(1.4)$ \\
\hline Asian, $n(\%)$ & $1(1.4)$ \\
\hline \multicolumn{2}{|c|}{ Ethnicity (excluding patients enrolled in France $[n=8]$} \\
\hline Hispanic or Latino, $n(\%)$ & $4(5.6)$ \\
\hline Not Hispanic or Latino, $n$ no. (\%) & $67(93.1)$ \\
\hline Unknown, $n$ no. $(\%)$ & $1(1.4)$ \\
\hline Variant hairy cell leukemia-no. (\%) & $3(3.8)$ \\
\hline Splenectomy-no. $(\%)$ & $5(6.3)$ \\
\hline \multicolumn{2}{|c|}{ Eastern Cooperative Oncology Group performance status-no. (\%) } \\
\hline 0 & $49(61.3)$ \\
\hline 1 & $29(36.3)$ \\
\hline 2 & $2(2.5)$ \\
\hline \multicolumn{2}{|l|}{ Extent of HCL } \\
\hline Median hemoglobin (range), $g / d L$ & $\begin{array}{l}11.10(6.5 \\
16.3)\end{array}$ \\
\hline Median neutrophil count (range), $n L$ & $0.81(0.1,6.2)$ \\
\hline Median platelet count (range), $n L$ & $68(6,350)$ \\
\hline $\begin{array}{l}\text { Median hairy cell involvement in bone marrow, } \\
\%(\text { (range) })^{\mathrm{a}}\end{array}$ & $85(0,100)$ \\
\hline $\begin{array}{l}\text { Median size of spleen, excluding splenectomy, } \\
\text { cm (range) }\end{array}$ & $13.3(8.9,24.7)$ \\
\hline \multicolumn{2}{|l|}{ Prior cancer therapy } \\
\hline Median number of lines of prior therapy (range) & $3.0(2,11)$ \\
\hline$>3$ prior lines, $n(\%)$ & $39(48.8)$ \\
\hline Prior purine nucleoside analog, $n(\%)$ & $80(100)$ \\
\hline Prior rituximab, $n(\%)$ & $60(75.0)$ \\
\hline
\end{tabular}

${ }^{a}$ Determined by blinded independent pathologist read of hematoxylin and eosin stained slides. Two patients were reported as not having baseline bone marrow involvement. In one case there were technical problems with the hematoxylin and eosin stained slides but $90 \%$ involvement by immunohistochemistry; in the other case the patient had variant HCL presenting as splenomegaly $(182 \mathrm{~mm})$

\section{Results}

\section{Patients}

The database was locked on 24 May 2017. Eighty patients were enrolled and treated (Table 1); $50(62.5 \%)$ patients completed six cycles of treatment and $12(15.0 \%)$ discontinued early after achieving complete response with negative minimal residual disease (Figure S1 in Supplementary Appendix). Enrolled patients had received a median of three lines of prior therapy; 70 patients $(87.5 \%)$ received at least two lines of purine nucleotide analogs, 60 
a
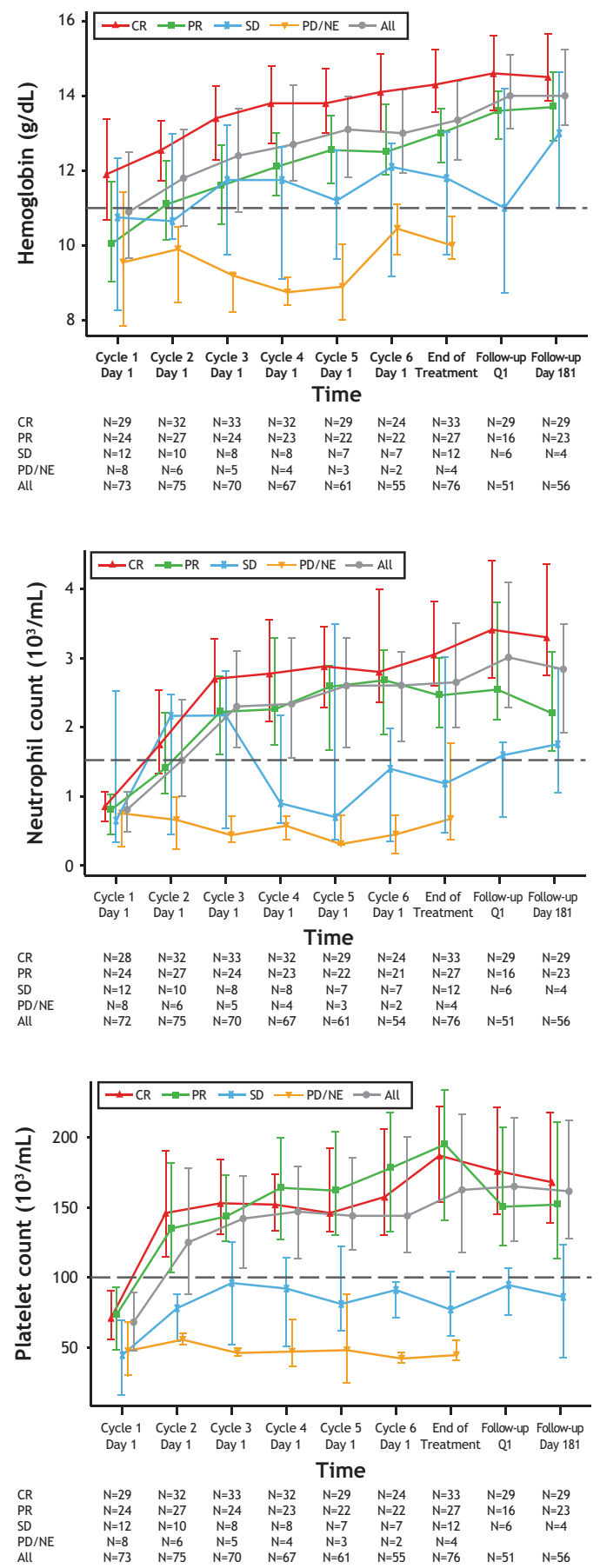

b
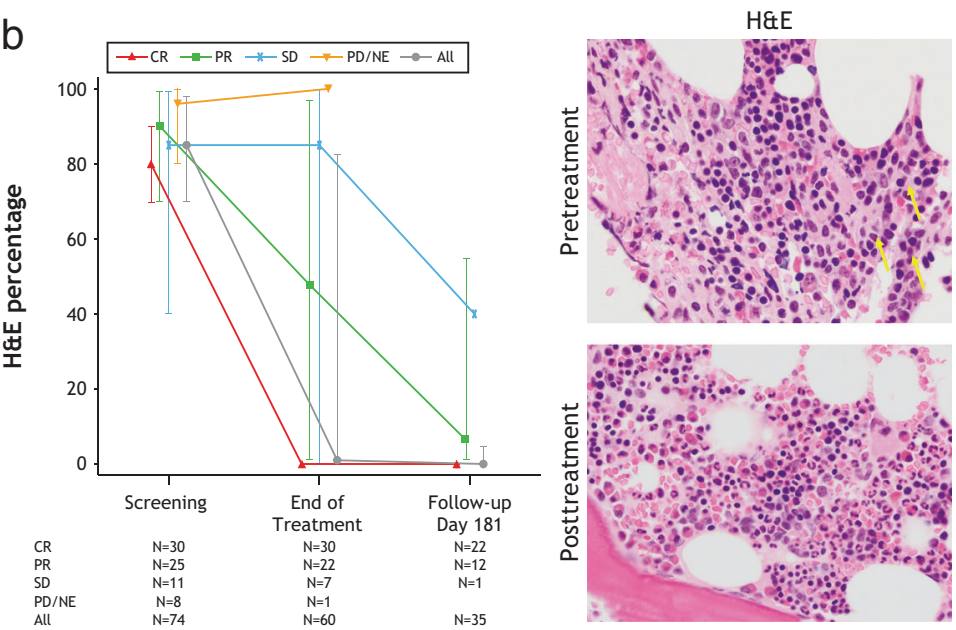

C

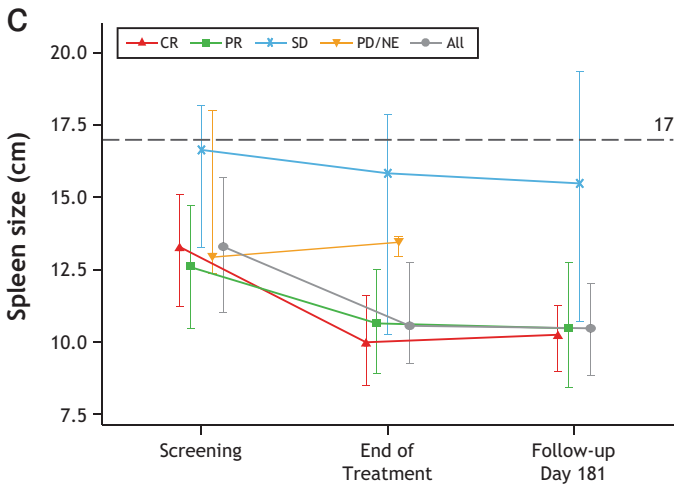

d

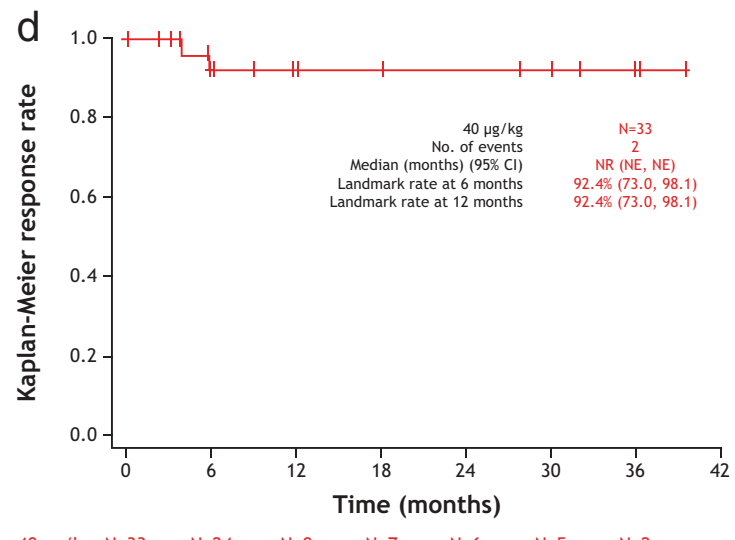

$40 \mu g / k g \quad N=33 \quad N=24 \quad N=9 \quad N=7 \quad N=6 \quad N=5 \quad N=2$

Fig. 1 Evaluation of primary end point (blinded independent central review). a Hemoglobin (top), neutrophil counts (middle), and platelet counts (bottom) over time, as a function of best objective response. Median and interquartile range are shown at each displayed time point and the threshold for hematologic remission is indicated as a dotted line. b HCL involvement in bone marrow, assessed by hematoxylin and eosin stain, as a function of best objective response; median and interquartile range are shown (top), and as representative pretreatment and posttreatment images $(\times 100)$ of a patient who obtained a minimal residual disease-negative complete response (bottom). Yellow arrows indicate hairy cells. c Spleen size by imaging, as a function of best objective response. Median and interquartile range are shown. Splenectomy patients $(n=5)$ not included. d Kaplan-Meier plot of duration of hematologic remission from complete response 
Table 2 Disease response and minimal residual disease status by immunohistochemistry

\begin{tabular}{|c|c|c|}
\hline \multirow[t]{2}{*}{ Parameter } & \multicolumn{2}{|l|}{ Value $(N=80)$} \\
\hline & $\begin{array}{l}\text { Blinded independent central } \\
\text { review }\end{array}$ & Investigator assessment \\
\hline $\begin{array}{l}\text { Durable complete response (primary end } \\
\text { point), } n(\%)^{\mathrm{a}}\end{array}$ & $24(30.0)$ & $38(47.5)$ \\
\hline $95 \%$ confidence interval & $20.3,41.3$ & $36.2,59.0$ \\
\hline \multicolumn{3}{|l|}{ Best overall response $^{a}$} \\
\hline Complete response, $n(\%)$ & $33(41.3)$ & $41(51.3)$ \\
\hline $95 \%$ confidence interval & $30.4,52.8$ & $39.8,62.6$ \\
\hline $\begin{array}{l}\text { Complete response, minimal residual } \\
\text { disease negative, } n(\%)\end{array}$ & $27(33.8)$ & $26(32.5)$ \\
\hline $95 \%$ confidence interval & $23.6,45.2$ & $22.4,43.9$ \\
\hline Partial response, $n(\%)$ & $27(33.8)$ & $27(33.8)$ \\
\hline $\begin{array}{l}\text { Objective response rate (complete or partial } \\
\text { response), } n(\%)^{\mathrm{a}}\end{array}$ & $60(75.0)$ & $63(78.8)$ \\
\hline $95 \%$ confidence interval & $64.1,84.0$ & $68.2,87.1$ \\
\hline
\end{tabular}

${ }^{a}$ Two-sided confidence interval was calculated using the exact probability method based on the binomial distribution patients $(75.0 \%)$ received prior rituximab and 14 patients (17.5\%) received prior BRAF-inhibitor (Table S1 in Supplementary Appendix).

\section{Efficacy}

Median hemoglobin, neutrophil count, and platelet count improved rapidly during treatment (Fig. 1a); $80 \%$ of patients (64/80) achieved hematologic remission in about one month (median 1.1 months, $95 \%$ CI, 1.0 to 1.2 ). At a median follow-up of 16.7 months [2.1 to 48.8]), the durable complete response rate was 30\% (24/80 patients; $95 \% \mathrm{CI}$, 20.3 to 41.3 ), and the objective response rate was $75 \%$ (60/ 80 patients, 95\% CI, 64.1 to 84.0 ; Table 2) based on blinded independent central review. The complete response rate was $41 \%$ (33/80 patients; 95\% CI, 30.4 to 52.8). Thirty-three patients achieved complete response, including elimination of leukemic cells in bone marrow by morphologic assessment; significant $(>90 \%)$ reductions in bone marrow involvement were also observed in $29.6 \%$ of patients achieving partial response (8/27; Fig. 1b). Average spleen size decreased during treatment, and among patients with baseline splenomegaly (17 $\mathrm{cm}$ or larger), 6 (42.9\%) resolved to $14 \mathrm{~cm}$ or smaller (Fig. 1c).

Most (28/33) complete responses were achieved at the end of treatment disease assessment; five patients achieved complete response at disease assessment six months after the end of treatment. The median duration of hematologic remission from complete response (Fig. 1d), median duration of complete response, and median progression-free survival were not reached. Six patients relapsed from complete response as of the data cut-off; four had asymptomatic relapse with only reappearance of hairy cells in the bone marrow with normal hematological counts and two had loss of hematologic remission. Among complete responders, $27(85 \%)$ patients achieved minimal residual disease negativity as assessed by immunohistochemistry (Table 2 and Fig. 2). The median duration of complete response for minimal residual disease-positive patients was 5.9 months and was not reached for minimal residual disease-negative patients (Fig. 2b). Subgroup analyses are presented in Figure S2 in the Supplementary Appendix.

\section{Safety}

The most common adverse events were peripheral edema $(38.8 \%)$, nausea (35.0\%), and fatigue $(33.8 \%)$ (Table 3$)$; the most common treatment-related adverse events were nausea $(27.5 \%)$, peripheral edema (26.3\%), headache $(21.3 \%)$, and pyrexia (20.0\%). The most common treatment-related grade $3 / 4$ adverse events were decreased lymphocyte count $(7.5 \%)$ and hemolytic uremia syndrome (5.0\%). Grade 3 or 4 infections occurred in $13(16.3 \%)$ patients, with infections in 2 patients $(2.5 \%)$ reported as treatment related. Serious adverse events in at least $5 \%$ of patients were hemolytic uremic syndrome (7.5\%), pyrexia (6.3\%), and capillary leak syndrome $(5.0 \%)$. Three deaths occurred on study due to pneumonia, septic shock, and sepsis syndrome and underlying HCL; none were considered treatment related. The most common treatment-related adverse events leading to permanent discontinuation were hemolytic uremic syndrome $(n=4)$, capillary leak syndrome $(n=2)$, and blood creatinine increased ( $n=2$; associated with hemolytic uremic syndrome). All hemolytic uremic syndrome and capillary leak syndrome events were reversible. Details 
Fig. 2 Assessment of minimal residual disease by

immunohistochemistry (blinded independent central review).

a Representative

immunohistochemistry images

from pretreatment and

posttreatment bone marrow

biopsy specimens of the same patient shown in Fig. 1b: CD20 (left) and PAX5/TRAP (right).

b Kaplan-Meier plot of duration of complete response, by

minimal residual disease status a
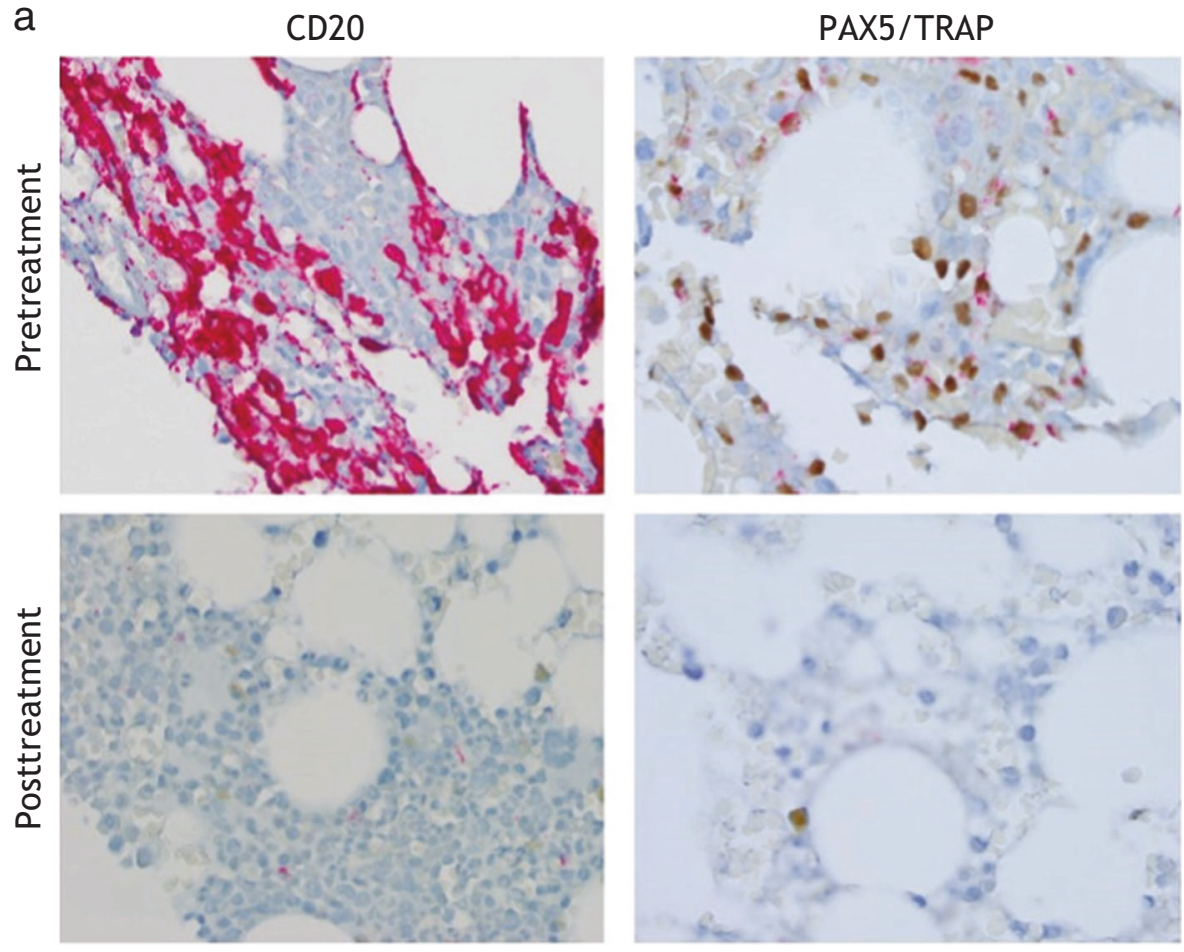

b

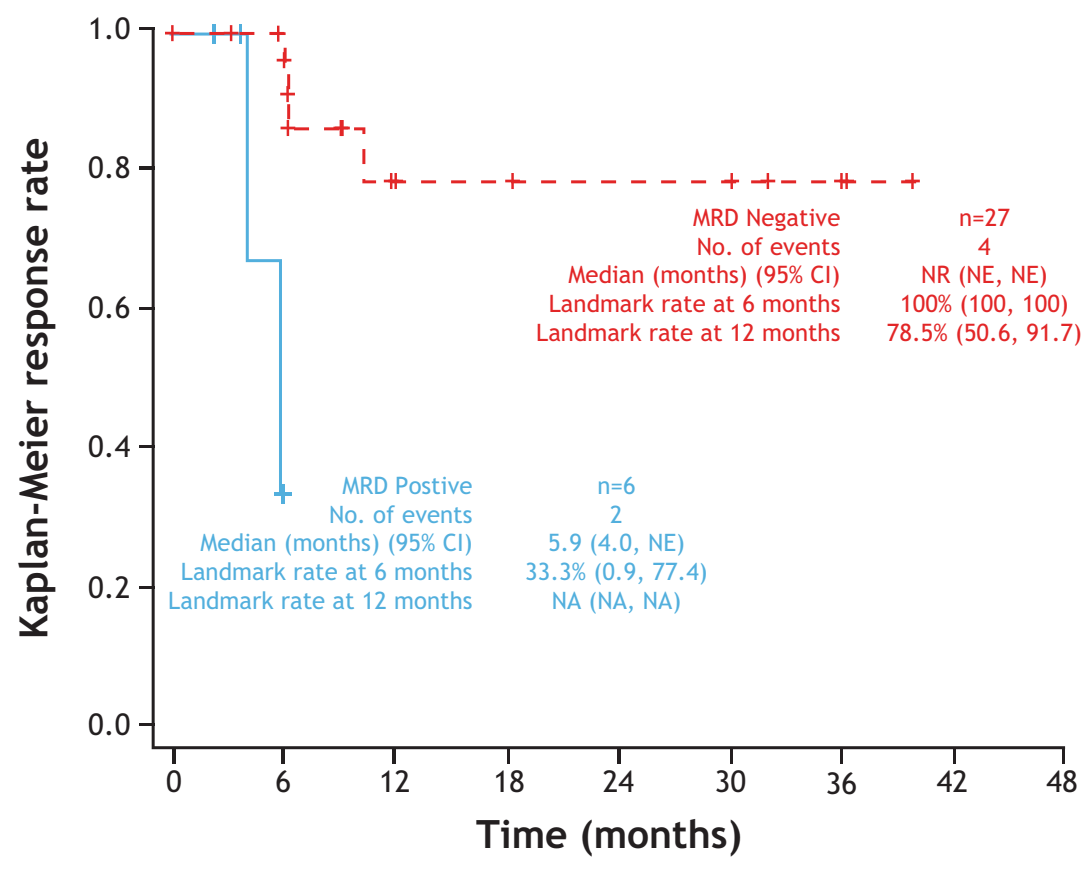

MRD Positive $\quad \mathrm{N}=6 \quad \mathrm{~N}=1$

MRD Negative $\mathrm{N}=27 \quad \mathrm{~N}=23$

$N=7 \quad N=6 \quad N=5 \quad N=5 \quad N=2$

regarding hemolytic uremic syndrome/capillary leak syndrome cases are presented in the Supplementary Appendix. Key laboratory findings are presented in Table S2. The median key immunoglobulin (IgA, IgG, and IgM) levels remained unchanged after treatment. Median CD4 T cell counts were stable or increased following a transient decrease on day eight.
Pharmacokinetics, immunogenicity, and pharmacodynamics

Moxetumomab pasudotox pharmacokinetics were characterized by rapid plasma concentration decline following intravenous administration (Table S3 and Figure S3A in Supplementary Appendix). Exposure was higher after 
Table 3 Summary of adverse events ${ }^{\mathrm{a}}$

\begin{tabular}{|c|c|c|}
\hline Adverse event & $\begin{array}{l}\text { All grades } \\
\text { Patients, } n(\%)\end{array}$ & Grades $3 / 4$ \\
\hline Edema peripheral & $31(38.8 \%)$ & 0 \\
\hline Nausea & $28(35.0 \%)$ & $2(2.5 \%)$ \\
\hline Fatigue & $27(33.8 \%)$ & 0 \\
\hline Headache & $26(32.5 \%)$ & 0 \\
\hline Pyrexia & $25(31.3 \%)$ & $1(1.3 \%)$ \\
\hline Hypocalcaemia & $19(23.8 \%)$ & 0 \\
\hline Hypophosphatemia & $19(23.8 \%)$ & $8(10.0 \%)$ \\
\hline Constipation & $18(22.5 \%)$ & 0 \\
\hline Anemia & $17(21.3 \%)$ & $8(10.0 \%)$ \\
\hline Diarrhea & $17(21.3 \%)$ & 0 \\
\hline Alanine aminotransferase increased & $17(21.3 \%)$ & $1(1.3 \%)$ \\
\hline Lymphocyte count decreased & $16(20.0 \%)$ & $16(20.0 \%)$ \\
\hline Hypoalbuminemia & $16(20.0 \%)$ & 0 \\
\hline Hypokalemia & $13(16.3 \%)$ & $2(2.5 \%)$ \\
\hline Hypertension & $12(15.0 \%)$ & $6(7.5 \%)$ \\
\hline Platelet count decreased & $9(11.3 \%)$ & $5(6.3 \%)$ \\
\hline Hyponatremia & $9(11.3 \%)$ & $2(2.5 \%)$ \\
\hline White blood cell count decreased & $8(10.0 \%)$ & $7(8.8 \%)$ \\
\hline Capillary leak syndrome & $7(8.8 \%)$ & $2(2.5 \%)$ \\
\hline Upper respiratory infection & $7(8.8 \%)$ & $2(2.5 \%)$ \\
\hline Hemolytic uremic syndrome & $6(7.5 \%)$ & $4(5.0 \%)$ \\
\hline Neutrophil count decreased & $6(7.5 \%)$ & $5(6.3 \%)$ \\
\hline Febrile neutropenia & $5(6.3 \%)$ & $4(5.0 \%)$ \\
\hline Neutropenia & $4(5.0 \%)$ & $4(5.0 \%)$ \\
\hline Hypoxia & $4(5.0 \%)$ & $2(2.5 \%)$ \\
\hline Lung infection & $3(3.8 \%)$ & $2(2.5 \%)$ \\
\hline Acute kidney injury & $3(3.8 \%)$ & $2(2.5 \%)$ \\
\hline Erysipelas & $2(2.5 \%)$ & $2(2.5 \%)$ \\
\hline
\end{tabular}

${ }^{a}$ Adverse events of any grade with an incidence of at least $20 \%$, as well as events of grade 3 or grade 4 with an incidence of at least $2.5 \%$

subsequent doses versus the first dose, likely related to treatment-mediated reduction of the CD22 sink (Figure S3B in Supplementary Appendix). Anti-drug antibodies were detected at baseline in 45/76 evaluable patients (59.2\%). The frequency of neutralizing antibodies and anti-drug antibody titer increased with repeated cycles of treatment; reduced drug exposure was observed in patients with hightiter (>10 000) anti-drug antibodies (Figures S3C and S3D in Supplementary Appendix). Median peripheral blood CD19 B cell counts (including normal B cells and hairy cells) were reduced by $90 \%$ on day eight, remained low through the end of treatment, and, for patients with partial or complete response, returned to approximately normal levels at six months posttreatment (Figure S3E in Supplementary Appendix).

\section{Discussion}

To date, this pivotal study is the largest prospective study in third-line or beyond relapsed/refractory HCL; it is also the first study using durable complete response (defined as complete response assessed by blinded independent central review with maintenance of hematologic remission for more than 180 days) as the primary end point. Patients were heavily pretreated; $50 \%$ received three or more prior courses of purine nucleoside analogs and $75 \%$ received prior rituximab. The blinded independent central review-assessed complete response rate of $41 \%$ represents a substantial improvement over historical controls, and the durable complete response rate of $30 \%$ met the primary end point. In HCL, immunohistochemistry staining of a high-quality bone marrow biopsy may provide more consistent minimal residual disease evaluation than flow cytometry of bone marrow aspirates, which depend on a consistent cellular yield [8]. Published data suggest that the extent of minimal residual disease remaining after therapy in HCL is important in predicting long-term outcome [23, 24]. Retrospective analysis of the phase 1 study of moxetumomab pasudotox in a similar patient population of relapsed/refractory HCL showed that patients who were minimal residual diseasenegative by immunohistochemistry had extended duration of response (82.7 versus 54.7 months). This result supported the possible predictive value of immunochemistrybased minimal residual disease for long-term outcome. In this study, a majority of patients (27/33) who achieved complete response also achieved negative minimal residual disease by immunohistochemistry. While long-term followup is ongoing, the fact that the median duration of complete response was reached for minimal residual disease-positive patients (5.9 months; with limitation of small patient numbers) but has not for minimal residual disease-negative patients indicates that long-term outcome may be improved by clearing minimal residual disease.

Consistent with prior studies of moxetumomab pasudotox and its predecessor molecule BL22 (CAT-3888) [25, 26], in this pivotal study, response rates were higher in patients who did not have splenomegaly or splenectomy (which was associated with high disease burden in the bone marrow; 4 of the 5 patients had $100 \%$ involvement at baseline). Three patients with HCL variant were enrolled; all had high disease burden (all had splenomegaly $>18 \mathrm{~cm}$ and two had lymphocytosis $>20 / \mathrm{nL}$ ) and did not achieve complete response. According to these observations, it may be advisable to initiate moxetumomab pasudotox treatment earlier in relapse when possible in patients with very high disease burden; further studies would be required to determine whether a more intensive treatment regimen might improve response rates in these patients. 
Substantial clinical activity was observed despite a high rate of immunogenicity; $\sim 75 \%$ of patients had detectable neutralizing antibodies at the end of treatment, regardless of response status. Patients who achieved complete or partial response typically maintained antibody titers below 10,000, and therefore maintained drug exposure for more treatment cycles than patients with stable or progressive disease, suggesting that approaches to reduce the immunogenicity of moxetumomab pasudotox might further improve response.

Hemolytic uremia syndrome and capillary leak syndrome were known toxicities and observed in the moxetumomab pasudotox phase 1 study and in studies of BL22 [25, 27]. In the current study, these events (observed in 10 patients [12.5\%]) were typically managed with close monitoring of vital signs and laboratory values (including blood pressure, body weight, blood creatinine, and schistocytes in peripheral blood smear) and supportive medical care (including adequate hydration), with intensive care without plasma exchange and treatment discontinuation for severe cases. Although the exact mechanisms are not well understood, our experience suggests that incidence and severity of hemolytic uremia syndrome and capillary leak syndrome may be reduced by ensuring adequate oral hydration during the first week of each cycle and proper (not excessive) intravenous fluid supplementation on the day of infusion. Dexamethasone may be considered in patients experiencing nausea or fever, to maintain oral hydration for adequate renal perfusion. Worsening renal function was observed in isolation and in association with hemolytic uremia syndrome; based on these findings, close monitoring of renal function is recommended during treatment.The pivotal study data demonstrate that moxetumomab pasudotox provides a deep and durable response with ability to eradicate minimal residual disease in a substantial fraction of patients with relapsed/refractory HCL who have exhausted available therapies, and has a favorable safety profile compared to other available agents. A high percentage of patients were able to receive the full treatment course, and the most important risks, hemolytic uremia syndrome, and capillary leak syndrome, although relatively infrequent, were manageable and reversible with close monitoring and best supportive care. Moxetumomab pasudotox treatment results in substantially less bone marrow suppression than purine nucleoside analogs, without exacerbating baseline infections. In conclusion, moxetumomab pasudotox offers a clinically meaningful treatment for patients with relapsed/refractory HCL.

\section{Data availability}

The data sets generated and/or analyzed during the current study are available from the corresponding author upon reasonable request.
Acknowledgements This study was sponsored in part by the National Cancer Institute Intramural Program. We thank the patients and their families who participated in this study. We also thank Elad Sharon, $\mathrm{MD}, \mathrm{MPH}$, for study monitoring during the CTEP-sponsored portion of the study, Julie Feurtado, RN, for collecting and reporting a portion of the study data, Sangmin Lee and Jiří Mayer for serving as investigators during the study, all investigational site personnel, and the MedImmune team, including Jamie Freeman, Mark Lanasa, Monica Hernandez, and Carolyn Pendry. Medical writing and editorial assistance were provided by Amy Zannikos, PharmD, of Peloton Advantage, Parsippany, NJ, and were funded by MedImmune.

Author Contributions: FJG served as Chairman of the Study Scientific Monitoring Committee (SSMC) which included CD, JD, BTG, PIC, TR, GJR, G. Saglio, XT, and PLZ.RJK, IP, G. Saglio, GJR, NSY, SM, $\mathrm{PH}, \mathrm{NS}, \mathrm{KB}$, and FJG contributed to the study conception and design. RJK, CD, PLZ, JD, LK, TR, DEG, PIC, S. Dietrich, M. Gotic, LL, FO, G. Schiller, RS, LB, MB, KB, DAB, AC, S. Dinner, MD, BTG, M. Gobbi, AH, SL, FM, FR, PR, MR, TS, TT, XT, CAY, WW, and FJG were study investigators.RJK, CD, PLZ, JD, LK, TR, DEG, PIC, S Dietrich, M. Gotic, LL, FO, G Schiller, RS, LB, MB, KB, DAB, AC, S Dinner, MD, BTG, M. Gobbi, AH, SL, FM, FR, PR, MR, TS, TT, XT, CAY, WW and FJG provided patients for the study.NSY, SM, PH, $\mathrm{NS}$, and KB participated in the collection and assembly of data.All authors participated in data analysis and interpretation, manuscript preparation, and manuscript review and revisions. All authors approved the manuscript for submission. FJG served as study Principal Investigator and Chairman of the SSMC and had final responsibility for the decision to submit this manuscript for publication.

\section{Compliance with ethical standards}

Conflict of interest This study was sponsored by MedImmune. RJK received a research grant from MedImmune and is co-inventor of moxetumomab pasudotox, with the NIH holding the patent. CD has received personal fees from Gilead, Infinity, Janssen/Pharacyclics, MedImmune, Roche, and Sanofi Aventis, and has received clinical trial support from MedImmune. PLZ has served as an advisor for Bristol-Myers Squibb, Celgene, Gilead, Janssen, Merck, Roche, Servier, and Takeda. JD served on a data monitoring board for MedImmune, has received research grants from Janssen and Roche, and has received personal fees from Abbvie, Gilead, Janssen, and Roche. LK, DEG, M Gotic, LL, FO, RS, GS, SD, LB, CAY, AH, KB, MB, MG, AC, MD, SD, SL, MR, PR, TT, GS, GJR, XT, and WW have no conflicts to report. TR has received research grants from AstraZeneca. PLC has served as a speaker for Bristol-Myers Squibb, Incyte, Novartis, and Pfizer. DAB has received personal fees from Amgen, Novartis, and Teva, and his institution has received reimbursement for clinical trials from Ablynx, Amgen, Astellas, Astex, Cyclacel, Genzyme, Janssen, MedImmune, Merus, Menarini, Novella Clinical, Pfizer, and Seattle Genetics. BTG has received personal fees from BerGenBio AS, Novartis AS, Pfizer, and Seattle Genetics, received non-financial support from Merck Sharpe \& Dohme and Roche, and owns shares in Alden Cancer Therapeutics 2 AS and Kinn Therapeutics AS. FM has received research grants from Amgen and Sandoz, received travel accommodations from Octapharma and Roche, and served on advisory boards for Pfizer, Roche, and Sandoz. FR has received research grants from MedImmune. TS has served on speakers' bureaus for Pharmacyclics and Seattle Genetics, and on a steering committee for Juno. KB, NS, PH, SM, and NSY are employees of MedImmune and may own stock/options in AstraZeneca. IP is co-inventor of moxetumomab pasudotox, with the NIH holding the patent. FG has received a research grant from MedImmune. 
Open Access This article is licensed under a Creative Commons Attribution 4.0 International License, which permits use, sharing, adaptation, distribution and reproduction in any medium or format, as long as you give appropriate credit to the original author(s) and the source, provide a link to the Creative Commons license, and indicate if changes were made. The images or other third party material in this article are included in the article's Creative Commons license, unless indicated otherwise in a credit line to the material. If material is not included in the article's Creative Commons license and your intended use is not permitted by statutory regulation or exceeds the permitted use, you will need to obtain permission directly from the copyright holder. To view a copy of this license, visit http://creativecommons. org/licenses/by/4.0/.

\section{References}

1. Grever MR, Blachly JS, Andritsos LA. Hairy cell leukemia: update on molecular profiling and therapeutic advances. Blood Rev. 2014;28:197-203.

2. Rosenberg JD, Burian C, Waalen J, Saven A. Clinical characteristics and long-term outcome of young hairy cell leukemia patients treated with cladribine: a single-institution series. Blood. 2014;123:177-83.

3. Kreitman RJ. Immunoconjugates and new molecular targets in hairy cell leukemia. Hematol Am Soc Hematol Educ Program. 2012;2012:660-6.

4. Thompson PA, Ravandi F. How I manage patients with hairy cell leukaemia. Br J Haematol. 2017;177:543-56.

5. Else M, Dearden CE, Matutes E, Garcia-Talavera J, Rohatiner AZ, Johnson SA, et al. Long-term follow-up of 233 patients with hairy cell leukaemia, treated initially with pentostatin or cladribine, at a median of 16 years from diagnosis. $\mathrm{Br} \mathrm{J}$ Haematol. 2009; 145:733-40.

6. Getta BM, Park JH, Tallman MS. Hairy cell leukemia: past, present and future. Best Pract Res Clin Haematol. 2015;28:269-72.

7. Zinzani PL, Pellegrini C, Stefoni V, Derenzini E, Gandolfi L, Broccoli A, et al. Hairy cell leukemia: evaluation of the long-term outcome in 121 patients. Cancer. 2010;116:4788-92.

8. Grever MR, Abdel-Wahab O, Andritsos LA, Banerji V, Barrientos J, Blachly JS, et al. Consensus guidelines for the diagnosis and management of patients with classic hairy cell leukemia. Blood. 2017;129:553-60.

9. Tiacci E, Park JH, De Carolis L, Chung SS, Broccoli A, Scott S, et al. Targeting mutant BRAF in relapsed or refractory hairy-cell leukemia. N Engl J Med. 2015;373:1733-47.

10. Hagberg $H$, Lundholm L. Rituximab, a chimaeric anti-CD20 monoclonal antibody, in the treatment of hairy cell leukaemia. $\mathrm{Br}$ J Haematol. 2001;115:609-11.

11. Lauria F, Lenoci M, Annino L, Raspadori D, Marotta G, Bocchia M, et al. Efficacy of anti-CD20 monoclonal antibodies (Mabthera) in patients with progressed hairy cell leukemia. Haematologica. 2001;86:1046-50.

12. Nieva J, Bethel K, Saven A. Phase 2 study of rituximab in the treatment of cladribine-failed patients with hairy cell leukemia. Blood. 2003;102:810-3.

13. Thomas DA, O'Brien S, Bueso-Ramos C, Faderl S, Keating MJ, Giles FJ, et al. Rituximab in relapsed or refractory hairy cell leukemia. Blood. 2003;102:3906-11.
14. Angelopoulou MK, Pangalis GA, Sachanas S, Kokoris SI, Anargyrou K, Galani Z, et al. Outcome and toxicity in relapsed hairy cell leukemia patients treated with rituximab. Leuk Lymphoma. 2008;49:1817-20.

15. Ravandi F, Jorgensen JL, O'Brien SM, Verstovsek S, Koller CA, Faderl S, et al. Eradication of minimal residual disease in hairy cell leukemia. Blood. 2006;107:4658-62.

16. Ravandi F, O'Brien S, Jorgensen J, Pierce S, Faderl S, Ferrajoli A, et al. Phase 2 study of cladribine followed by rituximab in patients with hairy cell leukemia. Blood. 2011;118:3818-23.

17. Burotto M, Stetler-Stevenson M, Arons E, Zhou H, Wilson W, Kreitman RJ. Bendamustine and rituximab in relapsed and refractory hairy cell leukemia. Clin Cancer Res. 2013; 19:6313-21.

18. Jones J, Andritsos L, Kreitman RJ, Ravandi F, Schiffer C, Call TG, et al. Efficacy and safety of the bruton tyrosine kinase inhibitor ibrutinib in patients with hairy cell leukemia: stage 1 results of a phase 2 study [abstract]. Blood. 2016;128:1215.

19. Kreitman RJ, Pastan I. Antibody fusion proteins: anti-CD22 recombinant immunotoxin moxetumomab pasudotox. Clin Cancer Res. 2011;17:6398-405.

20. Kreitman RJ, Tallman MS, Robak T, Coutre S, Wilson WH, Stetler-Stevenson M, et al. Minimal residual hairy cell leukemia eradication with moxetumomab pasudotox: phase I results and long-term follow-up. Blood. 2018;131(21):2331-4.

21. Kreitman JR, Dearden C, Zinzani P, Delgado J, Karlin L, Robak $\mathrm{T}$, et al. Moxetumomab pasudotox induces high rates of durable complete response and eradication of minimal residual disease in heavily pretreated patients with relapsed/refractory hairy cell leukemia: results of a pivotal international study (abstract). 59th Annual Meeting of the American Society of Hematology; 2017; Atlanta, GA.

22. Divino V, Karve S, Gaughan A, DeKoven M, Gao G, Knopf KB, et al. Characteristics and treatment patterns among US patients with hairy cell leukemia: a retrospective claims analysis. J Comp Eff Res. 2017;6:497-508.

23. Tallman MS. Implications of minimal residual disease in hairy cell leukemia after cladribine using immunohistochemistry and immunophenotyping. Leuk Lymphoma. 2011;52(suppl 2):65-8.

24. Mhawech-Fauceglia P, Oberholzer M, Aschenafi S, Baur A, Kurrer M, Von Rohr A, et al. Potential predictive patterns of minimal residual disease detected by immunohistochemistry on bone marrow biopsy specimens during a long-term follow-up in patients treated with cladribine for hairy cell leukemia. Arch Pathol Lab Med. 2006;130:374-7.

25. Kreitman RJ, Tallman MS, Robak T, Coutre S, Wilson WH, Stetler-Stevenson $\mathrm{M}$, et al. Phase I trial of anti-CD22 recombinant immunotoxin moxetumomab pasudotox (CAT-8015 or HA22) in patient with hairy cell leukemia. J Clin Oncol. 2012;30:1822-8.

26. Kreitman RJ, Stetler-Stevenson M, Margulies I, Noel P, Fitzgerald DJ, Wilson WH, et al. Phase II trial of recombinant immunotoxin RFB4(dsFv)-PE38 (BL22) in patients with hairy cell leukemia. J Clin Oncol. 2009;27:2983-90.

27. Kreitman RJ, Wilson WH, Bergeron K, Raggio M, StetlerStevenson M, FitzGerald DJ, et al. Efficacy of the anti-CD22 recombinant immunotoxin BL22 in chemotherapy-resistant hairycell leukemia. N Engl J Med. 2001;345:241-7. 


\section{Affiliations}

Robert J. Kreitman ${ }^{1} \cdot$ Claire Dearden $^{2} \cdot$ Pier Luigi Zinzani ${ }^{3} \cdot$ Julio Delgado $^{4} \cdot$ Lionel Karlin $^{5} \cdot$ Tadeusz Robak $^{6}$. Douglas E. Gladstone $\mathbb{D}^{7} \cdot$ Philipp le Coutre ${ }^{8} \cdot$ Sascha Dietrich $^{9} \cdot$ Mirjana Gotic $^{10}{ }^{10} \cdot$ Loree Larratt $^{11} \cdot$ Fritz Offner $^{12}$. Gary Schiller $^{13} \cdot$ Ronan Swords $^{14} \cdot$ Larry Bacon $^{15} \cdot$ Monica Bocchia ${ }^{16} \cdot$ Krimo Bouabdallah $^{17}$ - Dimitri A. Breems ${ }^{18}$. Agostino Cortelezzi ${ }^{19}$. Shira Dinner ${ }^{20} \cdot$ Michael Doubek $^{21} \cdot$ Bjorn Tore Gjertsen $\mathbb{1}^{22} \cdot$ Marco Gobbi $^{23}$. Andrzej Hellmann ${ }^{24}$. Stephane Lepretre ${ }^{25}$. Frederic Maloisel ${ }^{26}$. Farhad Ravandi ${ }^{27} \cdot$ Philippe Rousselot $^{28,29}$. Mathias Rummel $^{30} \cdot$ Tanya Siddiqi $^{31} \cdot$ Tamar Tadmor $^{32} \cdot$ Xavier Troussard $^{33}$. Cecilia Arana $\mathrm{Yi}^{34}$. Giuseppe Saglio ${ }^{35}$. Gail J. Roboz ${ }^{36} \cdot$ Kemal Balic $^{37} \cdot$ Nathan Standifer $^{37} \cdot$ Peng He $^{38} \cdot$ Shannon Marshall ${ }^{38} \cdot$ Wyndham Wilson $^{1}$. Ira Pastan ${ }^{1} \cdot$ Nai-Shun $\mathrm{Yao}^{38} \cdot$ Francis Giles ${ }^{39}$

1 National Cancer Institute, National Institutes of Health, Bethesda, MD, USA

2 The Royal Marsden NHS Foundation Trust, London, UK

3 Institute of Hematology, Seràgnoli University of Bologna, Bologna, Italy

4 Hospital Clinic, Barcelona, Spain

5 Centre Hospitalier Lyon Sud, Pierre-bénite, France

6 Medical University of Lodz, Copernicus Memorial Hospital, Lodz, Poland

7 Johns Hopkins Kimmel Cancer Center, Baltimore, MD, USA

8 Charité Universitätsmedizin, Berlin, Germany

9 Universitätsklinikum Heidelberg, Heidelberg, BadenWürttemberg, Germany

10 Clinical Center of Serbia, Belgrade, Serbia

11 University of Alberta, Edmonton, Alberta, Canada

12 Ghent University Hospital, Ghent, Belgium

13 David Geffen School of Medicine, UCLA, Los Angeles, CA, USA

14 Sylvester Comprehensive Cancer Center, University of Miami, Miami, FL, USA

15 St. James's Hospital, Dublin, Ireland

16 Azienda Ospedaliera Universitaria, University of Siena, Siena, Italy

17 Service d'hématologie, CHU Bordeaux, F-33000 Bordeaux, France

18 Ziekenhuis Netwerk Antwerpe, Antwerp, Belgium

19 Fondazione IRCCS Ca' Granda Ospedale Maggiore Policlinico, University of Milan, Milan, Italy

20 Northwestern Medicine Feinberg School of Medicine,
Chicago, IL, USA

21 Masaryk University, Brno, Czech Republic

22 Helse Bergen HF Haukeland University Hospital, Bergen, Norway

23 Clinic of Hematology, Ospedale Policlinico San Martino, Genova, Italy

24 Department of Hematology and Transplantation, Medical University of Gdańsk, Gdańsk, Poland

25 Inserm U1245 and Department of Hematology, Centre Henri Becquerel and Normandie Univ UNIROUEN, Rouen, France

26 SOL, Clinique Sainte-Anne, Strasbourg, France

27 MD Anderson Cancer Center, Houston, TX, USA

28 Centre Hospitalier de Versailles, INSERM U1173 Le Chesnay, France

29 Université Versailles Saint-Quentin-en-Yvelines, Paris Saclay, France

30 Justus-Liebig University, Giessen, Germany

31 City of Hope National Medical Center, Duarte, CA, USA

32 Bnai Zion Medical Center, Haifa, Israel

33 L'hôpital Côte de Nacre, Caen Cedex 9, Caen, France

34 University of New Mexico, Albuquerque, NM, USA

35 University of Turin, Turin, Italy

36 Weill Cornell Medicine, The New York Presbyterian Hospital, New York, NY, USA

37 MedImmune, South San Francisco, CA, USA

38 MedImmune, Gaithersburg, MD, USA

39 Developmental Therapeutics Consortium, Chicago, IL, USA 